\title{
Spectral Properties of Homogeneous and Nonhomogeneous Radar Images
}

\section{Madsen, Søren Nørvang}

Published in:

I E E E Transactions on Aerospace and Electronic Systems

Link to article, DOI:

10.1109/TAES.1987.310891

Publication date:

1987

Document Version

Publisher's PDF, also known as Version of record

Link back to DTU Orbit

Citation (APA):

Madsen, S. N. (1987). Spectral Properties of Homogeneous and Nonhomogeneous Radar Images. I E E E Transactions on Aerospace and Electronic Systems, AES-23(4), 583-588.

https://doi.org/10.1109/TAES.1987.310891

\section{General rights}

Copyright and moral rights for the publications made accessible in the public portal are retained by the authors and/or other copyright owners and it is a condition of accessing publications that users recognise and abide by the legal requirements associated with these rights.

- Users may download and print one copy of any publication from the public portal for the purpose of private study or research.

- You may not further distribute the material or use it for any profit-making activity or commercial gain

- You may freely distribute the URL identifying the publication in the public portal

If you believe that this document breaches copyright please contact us providing details, and we will remove access to the work immediately and investigate your claim. 


\section{Spectral Properties of Homogeneous and Nonhomogeneous Radar Images}

\author{
SØREN NØRVANG MADSEN
}

Technical University of Denmark

On the basis of a two-dimensional, nonstationary white noise model for the complex radar backscatter, the spectral properties of a one-look synthetic-aperture radar (SAR) system is derived. It is shown that the power spectrum of the complex SAR image is scene independent. It is also shown that the spectrum of the intensity image is in general related to the radar scene spectrum by a linear integral equation, a Fredholm's integral equation of the third kind. Under simplifying assumptions, a closed-form equation giving the radar scene spectrum as a function of the SAR image spectrum can be derived.

\section{INTRODUCTION}

Synthetic aperture radar (SAR) is a technique that provides high resolution radar images. This is achieved through use of a coherent radar system and advanced signal processing [1].

The applications of SAR include sea ice mapping and monitoring, agricultural and geological investigations, oil spill detection, and more general oceanographic studies, and the list is rapidly increasing. In order to derive the most from the radar images, it is necessary to fully understand the peculiarities of radar images. One of the most important "artifacts" is the speckle effect. Since radar systems are bandpass systems operating on a microwave carrier frequency, it is found that the addition of several scatterers within one resolution cell will involve phase shifts due to different delays of the carrier [2]. The result is the speckle effect that makes the radar image of a homogeneous target field appear as a random process and the amplitude of the image signal is given by the Rayleigh distribution [3].

It has been reported that empirical distribution functions (derived from analysis of data windows) is nonRayleigh (e.g., [4]). The present model deals with the non-Rayleigh observations by modeling the scene as being a nonstationary white-noise process.

Speckle has a strong impact on the relation between spatial frequencies of the average backscatter coefficient $\sigma^{0}(x, y)$ (called the scene spectrum) and the spatial frequencies of the power detected SAR image (the image spectrum). The purpose of the paper is to present the analytical relation between the scene spectrum and the image spectrum, and thereby facilitate the inversion of an observed spectrum. Such an inversion is important, for instance, when SAR is applied to imaging ocean waves $[5,6]$. This application is mainly concerned with extracting the wavelength and direction of the ocean waves.

In this paper only complex images and one-look power detected images are considered. Furthermore, moving targets are excluded from the analysis. The models applied to the scene and the radar system are presented in Section II. In Section III a statistical description of radar images is given, including formulas for the power density spectrum of complex SAR images and one-look power detected images, respectively. In Section IV some simplifying assumptions are introduced, and the theory of the paper is compared with a previously used model.

\section{RADAR SCENE AND SAR SYSTEM MODELS}

\section{A. Radar Scene Model}

The model of a radar scene by necessity has to reflect the fact that reflections are superimposed on a complex signal basis, since both amplitude and phase are needed to calculate the reflection from a compound reflection [1]. 
A useful model is found by assuming that the scene consists of a set of point scatterers, so that the complex backscatter coefficient per area, $\sigma_{c}(\mathbf{r})=\sigma_{c}(x, y)$, can be written

$\sigma_{c}(\mathbf{r})=\sum_{l} \sqrt{\sigma_{l}} \exp \left(j \theta_{l}\right) \delta\left(\mathbf{r}-\mathbf{r}_{l}\right)$

where $\sigma_{l}$ is the radar cross section (RCS) of scatterer $l, \theta_{l}$ is the total phase shift of scatterer $l$ due to the reflection and the slant range delay, $\mathbf{r}_{l}$ is the position of scatterer $l$, and $\delta(\mathbf{r})$ is the two-dimensional Dirac delta function.

By introducing assumptions such as independence of the phasors, uniform distribution from 0 to $2 \pi$ of the phasors, and the presence of many scatterers within the radar impulse response (IPR) it can be justified [7] that $\sigma_{c}(\mathbf{r})$ is modeled as a white-noise complex circular symmetric Gaussian process with the autocorrelation function

$R_{\sigma_{c}}\left(\mathbf{r}_{1}, \mathbf{r}_{2}\right)=\delta\left(\mathbf{r}_{1}-\mathbf{r}_{2}\right) \sigma^{0}\left(\mathbf{r}_{2}\right)$.

For a homogeneous scene, which is equal to a spatially stationary process we find

$R_{\sigma_{c}}\left(\mathbf{r}_{1}, \mathbf{r}_{2}\right)=R_{\sigma_{c}}\left(\mathbf{r}_{1}-\mathbf{r}_{2}\right)=\sigma^{0} \delta\left(\mathbf{r}_{1}-\mathbf{r}_{2}\right)$

with the corresponding power density spectrum

$S_{\sigma_{c}}(\mathbf{f})=\int R_{\sigma_{c}}(\mathbf{r}) \exp (-j \pi \mathbf{r} \cdot \mathbf{f}) d \mathbf{r}=\sigma^{0}$.

\section{B. SAR System Model}

The problem of modeling a SAR system is many sided. First, the system is not invariant, which is generally required to define a frequency transfer function. Second, a SAR system involves numerous nonlinear mechanisms (e.g., aliasing, A/D conversion, processor), which cannot be included in a linear model. However, it can be argued, that the simple spatial invariant model shown in Fig. 1 will be useful $([7,8,9])$, where $\sigma_{c}(x, y)$ is the complex radar scene, $H_{e}\left(f_{x}, f_{y}\right)$ and $H_{c}\left(f_{x}, f_{y}\right)$ is the encoding and the compression transfer functions, and $n(x, y)$ is the thermal noise.

In the following we define:

$$
\begin{aligned}
H(\mathbf{f}) & =H\left(f_{x}, f_{y}\right)=H_{e}\left(f_{x}, f_{y}\right) \cdot H_{c}\left(f_{x}, f_{y}\right) \\
h(\mathbf{r}) & =h(x, y)=F^{-1}\left\{H\left(f_{x}, f_{y}\right)\right\} \\
& =\iint H\left(f_{x}, f_{y}\right) \exp \left[j 2 \pi\left(f_{x} x+f_{y} y\right)\right] d f_{x} d f_{y} .
\end{aligned}
$$

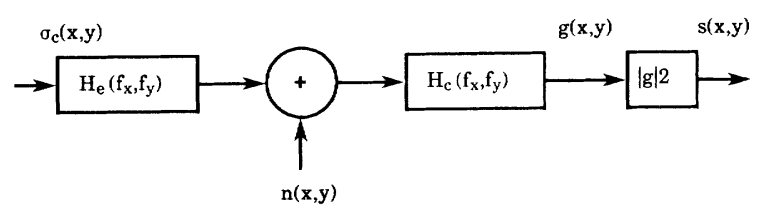

Fig. 1. Simplified spatially invariant SAR model, with power detection.

\section{STATISTICS OF RADAR IMAGERY}

The autocorrelation of the radar target scene is, from (2),

$R_{\sigma_{c}}\left(\mathbf{r}_{0}+\mathbf{r}, \mathbf{r}_{0}\right)=\delta(\mathbf{r}) \cdot \sigma^{0}\left(\mathbf{r}_{0}\right)$.

Within a target space, it is reasonable to assume that a spatial average value of the radar backscatter will exist, so that we have

$$
\begin{aligned}
\overline{\sigma^{0}} & =\left\langle\sigma^{0}\right\rangle \\
& =\lim _{X \rightarrow \infty} \lim _{Y \rightarrow \infty} \frac{1}{4 X Y} \int_{-X}^{X} \int_{-Y}^{Y} \sigma^{0}(x, y) d x d y .
\end{aligned}
$$

where the overbar and \langle\rangle indicate space averages. (In practice, the analyzed windows are always of finite extent, and the behaveor of $\sigma^{0}$ outside this window will be irrelevant.)

The spatial averaged correlation function (see Appendix A) is, from (7), (8), and (A6),

$\bar{R}_{\sigma_{c}}(\mathbf{r})=\overline{\sigma^{0}} \cdot \delta(\mathbf{r})$

and the corresponding average power spectrum is

$\bar{S}_{\sigma_{c}}(\mathbf{f})=\overline{\sigma^{0}}$.

Equations (9) and (10) show that even for nonhomogeneous (eventually periodic) scenes, the backscatter process is still 'white (in the sense that the power spectrum is constant as a function of frequency). From (A8) and (10) we find the spectrum of the complex radar image $g$,

$\bar{S}_{g}(\mathbf{f})=|H(\mathbf{f})|^{2} \overline{\sigma^{0}}$.

So, for nonstationary radar scenes the average spectrum is given by the power transfer function of the SAR system. Therefore, to estimate the power transfer function of a SAR system homogeneous areas are not required. The significant result is that the shape of the power transfer function of the complex SAR data will not depend on homogeneity of the scene. This is in contrast to the detected images, where the scene spectrum has a direct impact on the image spectrum as will be discussed below.

We shall start the analysis of the detected image $s$ by expressing the nonstationary autocorrelation function $R_{s}$ by the autocorrelation of the complex image $g$.

$$
\begin{aligned}
R_{s}\left(\mathbf{r}_{0}+\mathbf{r}, \mathbf{r}_{0}\right)= & E\left\{s\left(\mathbf{r}_{0}+\mathbf{r}\right) \cdot s\left(\mathbf{r}_{0}\right)\right\} \\
= & E\left\{g\left(\mathbf{r}_{0}+\mathbf{r}\right) g^{*}\left(\mathbf{r}_{0}+\mathbf{r}\right) g\left(\mathbf{r}_{0}\right) g^{*}\left(\mathbf{r}_{0}\right)\right\} \\
= & E\left\{g\left(\mathbf{r}_{0}+\mathbf{r}\right) g^{*}\left(\mathbf{r}_{0}+\mathbf{r}\right)\right\} \cdot E\left\{g\left(\mathbf{r}_{0}\right) g^{*}\left(\mathbf{r}_{0}\right)\right\} \\
& +E\left\{g\left(\mathbf{r}_{0}+\mathbf{r}\right) g^{*}\left(\mathbf{r}_{0}\right)\right\} \\
& \cdot E\left\{g^{*}\left(\mathbf{r}_{0}+\mathbf{r}\right) g\left(\mathbf{r}_{0}\right)\right\} \\
= & R_{g}\left(\mathbf{r}_{0}+\mathbf{r}, \mathbf{r}_{0}+\mathbf{r}\right) R_{g}\left(\mathbf{r}_{0}, \mathbf{r}_{0}\right) \\
& +\left|R_{g}\left(\mathbf{r}_{0}+\mathbf{r}, \mathbf{r}_{0}\right)\right|^{2}
\end{aligned}
$$


The third equality is based on a fundamental property of zero-mean complex Gaussian processes [10].

We can evaluate the individual terms of the right-hand side to be

$$
\begin{aligned}
R_{g}\left(\mathbf{r}_{0}+\mathbf{r}, \mathbf{r}_{0}+\mathbf{r}\right) & =\int|h(\mathbf{a})|^{2} \cdot \sigma^{0}\left(\mathbf{r}_{0}+\mathbf{r}-\mathbf{a}\right) d \mathbf{a} \\
R_{g}\left(\mathbf{r}_{0}, \mathbf{r}_{0}\right) & =\int|h(\mathbf{b})|^{2} \cdot \sigma^{0}\left(\mathbf{r}_{0}-\mathbf{b}\right) d \mathbf{b}
\end{aligned}
$$

and finally,

$$
R_{g}\left(\mathbf{r}_{0}+\mathbf{r}, \mathbf{r}_{0}\right)=\int h(\mathbf{a}) h^{*}(\mathbf{a}-\mathbf{r}) \boldsymbol{\sigma}^{0}\left(\mathbf{r}_{0}+\mathbf{r}-\mathbf{a}\right) d \mathbf{a}
$$

For a homogeneous area this gives

$$
\begin{aligned}
R_{s}(\mathbf{r})= & \left(\sigma^{0}\right)^{2}\left\{\left(\int|h(\mathbf{a})|^{2} d \mathbf{a}\right)^{2}\right. \\
& \left.+\left|\int h(\mathbf{a}) h^{*}(\mathbf{a}-\mathbf{r}) d \mathbf{a}\right|^{2}\right\} \\
= & \left(\sigma^{0}\right)^{2}\left\{R_{h}(\mathbf{0})^{2}+\left|R_{h}(\mathbf{r})\right|^{2}\right\}
\end{aligned}
$$

where $0=(0,0)$ and $R_{h}$ is defined by

$R_{h}(\mathbf{r})=\int h(\mathbf{a}) h *(\mathbf{a}-\mathbf{r}) d \mathbf{a}$.

The spectrum of a homogeneous area is then

$$
\begin{aligned}
S_{s}(\mathbf{f})= & \left(\boldsymbol{\sigma}^{0}\right)^{2}\left\{R_{h}(\mathbf{0})^{2} \delta(\mathbf{f})\right. \\
& \left.\left.+\int|H(\mathbf{f}+\mathbf{X})|^{2}|H(\mathbf{X})|^{2} d \mathbf{X}\right)\right\} .
\end{aligned}
$$

The first term is the dc component and the second term is the autocorrelation of the power transfer function. This shows that the spectrum of a homogeneous area is given if only the power transfer function $|H(\mathbf{f})|^{2}$ is known. However, (11) showed that the power transfer function can be obtained from the complex image $g$ without assuming stationarity.

Next we find in the nonstationary case

$$
\begin{aligned}
\bar{R}_{s}(\mathbf{r})= & \left\langle\iint|h(\mathbf{a})|^{2}|h(\mathbf{b})|^{2} \sigma^{0}\left(\mathbf{r}_{0}+\mathbf{r}-\mathbf{a}\right)\right. \\
& \times \sigma^{0}\left(\mathbf{r}_{0}-\mathbf{b}\right) d \mathbf{a} d \mathbf{b} \\
& +\iint h(\mathbf{a}) h^{*}(\mathbf{a}-\mathbf{r}) h^{*}(\mathbf{b}) h(\mathbf{b}-\mathbf{r}) \\
& \left.\times \sigma^{0}\left(\mathbf{r}_{0}+\mathbf{r}-\mathbf{a}\right) \sigma^{0}\left(\mathbf{r}_{0}+\mathbf{r}-\mathbf{b}\right) d \mathbf{a} d \mathbf{b}\right\rangle \\
= & \iint|h(\mathbf{a})|^{2}|h(\mathbf{b})|^{2} \bar{R}_{\mathbf{\sigma}^{0}}(\mathbf{r}+\mathbf{b}-\mathbf{a}) d \mathbf{a} d \mathbf{b} \\
& +\iint h(\mathbf{a}) h^{*}(\mathbf{a}-\mathbf{r}) h^{*}(\mathbf{b}) h(\mathbf{b}-\mathbf{r}) \\
& \times \bar{R}_{\mathbf{\sigma}^{0}}(\mathbf{b}-\mathbf{a}) d \mathbf{a} d \mathbf{b} .
\end{aligned}
$$

We now calculate the average spectrum

$$
\begin{aligned}
\bar{S}_{s}(\mathbf{f}) & =\int \bar{R}_{s}(\mathbf{r}) \exp (-j 2 \pi \mathbf{r} \cdot \mathbf{f}) d \mathbf{r} \\
& =\bar{S}_{1}(\mathbf{f})+\bar{S}_{2}(\mathbf{f}) .
\end{aligned}
$$

For convenience, we will transform the two terms on the right-hand side of (19) separately.

$$
\begin{aligned}
\bar{S}_{1}(\mathbf{f})= & \iiint|h(\mathbf{a})|^{2}|h(\mathbf{b})|^{2} \bar{R}_{\sigma^{0}}(\mathbf{r}+\mathbf{b}-\mathbf{a}) \\
& \times \exp (-j 2 \pi \mathbf{r} \cdot \mathbf{f}) d \mathbf{a} d \mathbf{b} d \mathbf{r} \\
= & \left.\left.S_{\sigma^{0}}(\mathbf{f}) \cdot\left|\int\right| h(\mathbf{a})\right|^{2} \exp (-j 2 \pi \mathbf{f} \cdot \mathbf{a}) d \mathbf{a}\right|^{2} \\
= & \bar{S}_{\sigma^{0}}(\mathbf{f}) \cdot \mathbf{T}(\mathbf{0}, \mathbf{f})
\end{aligned}
$$

where $\mathbf{T}$ is defined by

$$
\mathbf{T}(\mathbf{r}, \mathbf{f})=\left|\int h(\mathbf{a}) h^{*}(\mathbf{a}-\mathbf{r}) \exp (-j 2 \pi \mathbf{f} \cdot \mathbf{a}) d \mathbf{a}\right|^{2} .
$$

The second term of (19) can be expanded to

$$
\begin{aligned}
\bar{S}_{2}(\mathbf{f})= & \iiint \int h(\mathbf{a}) h^{*}(\mathbf{a}-\mathbf{r}) h^{*}(\mathbf{b}) h(\mathbf{b}-\mathbf{r}) \\
& \exp (-j 2 \pi \mathbf{f} \cdot \mathbf{r}) \bar{S}_{\sigma^{0}}\left(\mathbf{f}^{\prime}\right) \\
& \times \exp \left[j 2 \pi \mathbf{f}^{\prime}(\mathbf{b}-\mathbf{a})\right] d \mathbf{a} d \mathbf{b} d \mathbf{r} d \mathbf{f}^{\prime} \\
= & \iint \mathbf{T}\left(\mathbf{r}, \mathbf{f}^{\prime}\right) \cdot \bar{S}_{\sigma^{0}}\left(\mathbf{f}^{\prime}\right) \exp (-j 2 \pi \mathbf{f} \cdot \mathbf{r}) d \mathbf{r} d \mathbf{f}^{\prime} \\
= & \int \mathbf{T}^{\prime}\left(\mathbf{f}, \mathbf{f}^{\prime}\right) \bar{S}_{\sigma^{0}}\left(\mathbf{f}^{\prime}\right) d \mathbf{f}^{\prime}
\end{aligned}
$$

where

$\mathbf{T}^{\prime}\left(\mathbf{f}, \mathbf{f}^{\prime}\right)=\int \mathbf{T}\left(\mathbf{r}, \mathbf{f}^{\prime}\right) \cdot \exp (-j 2 \pi \mathbf{f} \cdot \mathbf{r}) d \mathbf{r}$.

In summary we find

$$
\bar{S}_{s}(\mathbf{f})=\bar{S}_{\sigma^{0}}(\mathbf{f}) \mathbf{T}(\mathbf{0}, \mathbf{f})+\int \mathbf{T}^{\prime}\left(\mathbf{f}, \mathbf{f}^{\prime}\right) \bar{S}_{\sigma^{0}}\left(\mathbf{f}^{\prime}\right) d \mathbf{f}^{\prime} .
$$

It is seen that to perform the conversion from $\bar{S}_{\sigma^{0}}(\mathbf{f})$ to $\bar{S}_{s}(\mathbf{f})$ we must know $\mathbf{T}(\mathbf{r}, \mathbf{f})$ from (22).

If we assume that the scene is homogeneous, (25) will be reduced to

$$
\begin{aligned}
\bar{S}_{s}(\mathbf{f})= & \left(\sigma^{0}\right)^{2} \delta(\mathbf{f}) \mathbf{T}(\mathbf{0}, \mathbf{0})+\left(\sigma^{0}\right)^{2} \mathbf{T}^{\prime}(\mathbf{f}, \mathbf{0}) \\
= & \left(\sigma^{0}\right)^{2}\{\mathbf{T}(\mathbf{0}, \mathbf{0}) \cdot \delta(\mathbf{f}) \\
& \left.+\int \mathbf{T}(\mathbf{r}, \mathbf{0}) \exp (-j 2 \pi \mathbf{f} \cdot \mathbf{r}) d \mathbf{r}\right\} .
\end{aligned}
$$

By inverse Fourier transformation we find

$\bar{R}_{s}(\mathbf{r})=\left(\sigma^{0}\right)^{2}\{\mathbf{T}(\mathbf{0}, \mathbf{0})+\mathbf{T}(\mathbf{r}, \mathbf{0})\}$

which checks with (16) and (17). It must be noted that only $\mathbf{T}(\mathbf{r}, \mathbf{0})$, and not $\mathbf{T}(\mathbf{r}, \mathbf{f})$ can be estimated from homogeneous targets. By expansion of $\mathbf{T}(\mathbf{0}, \mathbf{f})$ and $\mathbf{T}^{\prime}\left(\mathbf{f}, \mathbf{f}^{\prime}\right)$ one finds

$$
\begin{aligned}
\mathbf{T}(\mathbf{r}, \mathbf{f})= & \mid \int H(\zeta) H^{*}(\boldsymbol{\zeta}-\mathbf{f}) \\
& \times\left.\exp (j 2 \pi \mathbf{r} \cdot \zeta) d \zeta\right|^{2} \\
\mathbf{T}(\mathbf{0}, \mathbf{f})= & \left|\int H(\zeta) H^{*}(\boldsymbol{\zeta}-\mathbf{f}) d \zeta\right|^{2} \\
\mathbf{T}^{\prime}\left(\mathbf{f}, \mathbf{f}^{\prime}\right)= & \int H(\zeta+\mathbf{f}) H^{*}\left(\boldsymbol{\zeta}+\mathbf{f}-\mathbf{f}^{\prime}\right)
\end{aligned}
$$




$$
\times H^{*}(\boldsymbol{\zeta}) H\left(\boldsymbol{\zeta}-\mathbf{f}^{\prime}\right) d \zeta
$$

From these equations we observe that if the system is bandlimited, so that

$H(\mathbf{f})=H\left(f_{x}, f_{y}\right)=0$ for $\left|f_{x}\right| \geq \frac{B_{x}}{2} \quad$ or $\quad\left|f_{y}\right| \geq \frac{B_{y}}{2}$

then the integration bounds of (25) can be limited to $-B_{x}$ $\leqq f_{x} \leqq B_{x}$ and $-B_{y} \leqq f_{y} \leqq B_{y}$.

When finite integration intervals are introduced in (25), we find that the equation has the form of a Fredholm integral equation of the third kind. To solve (25) for $\bar{S}_{\sigma^{0}}(\mathbf{f})$ numerical methods must in general be applied. The approach to be used should depend on properties of the impulse response function $h(\mathbf{a})$ but also $\cdot \frac{\left[\sigma_{n} R_{h_{c}}(\mathbf{0})\right]^{2}+\int \bar{S}_{\sigma^{0}}(\mathbf{f}) \mathbf{T}(\mathbf{0}, \mathbf{f}) d \mathbf{f}+2 \sigma_{n} R_{h_{c}}(\mathbf{0}) \overline{\sigma^{0}} R_{h}(\mathbf{0})}{\left[\overline{\sigma^{0}} R_{h}(\mathbf{0})+\sigma_{n} R_{h_{c}}(\mathbf{0})\right]^{2}}$.

Therefore, given a backscatter spectrum $\bar{S}_{\sigma^{0}}(\mathbf{f})$ the

PMR varies with the signal to noise ratio. For pure noise PMR $=2$ and for pure signal

$\mathrm{PMR}_{\sigma^{0}}=2 \cdot \frac{\int S_{\sigma^{0}}(\mathbf{f}) T(\mathbf{0}, \mathbf{f}) d \mathbf{f}}{\left[\overline{\sigma^{0}} R_{h}(\mathbf{0})\right]^{2}}$

If $\mathrm{PMR}_{\sigma^{0}}$ is known, then (35) can in principle be used to estimate signal to noise ratio $S / N$ in an uncalibrated radar. We find from (35) and (36) that

$$
\mathrm{PMR}=2 \cdot \frac{1+\frac{\int S_{\sigma^{0}}(\mathbf{f}) T(\mathbf{0}, \mathbf{f}) d \mathbf{f}}{\left[\overline{\sigma^{0}} R_{h}(\mathbf{0})\right]^{2}}\left(\frac{\overline{\sigma^{2}} R_{h}(\mathbf{0})}{\sigma_{n} R_{h_{c}}(\mathbf{0})}\right)^{2}+2\left(\frac{\overline{\sigma^{0}} R_{h}(\mathbf{0})}{\sigma_{n} R_{h_{c}}(\mathbf{0})}\right)}{\left(1+\frac{\overline{\sigma^{0}} R_{h}(\mathbf{0})}{\sigma_{n} R_{h_{c}}(\mathbf{0})}\right)^{2}}
$$

on a priori knowledge of $\bar{S}_{\sigma^{0}}(\mathbf{f})$. The moment method [11] should be considered.

The inclusion of thermal noise in the analysis is straightforward though tedious. If we assume the thermal noise is stationary, that is, constant, and we furthermore use that thermal noise and signal are independent, then it can be shown that

$$
\begin{aligned}
\bar{S}_{s}(\mathbf{f})= & \left\{\sigma_{n}^{2} R_{h_{c}}(\mathbf{0})^{2}+2 \sigma_{n} \overline{\sigma^{0}} R_{h}(\mathbf{0}) R_{h_{c}}(\mathbf{0})\right\} \delta(\mathbf{f}) \\
& +\bar{S}_{\sigma^{0}}(\mathbf{f}) \mathbf{T}(\mathbf{0}, \mathbf{f})+\int \mathbf{T}^{\prime}\left(\mathbf{f}, \mathbf{f}^{\prime}\right) \bar{S}_{\sigma^{0}}\left(\mathbf{f}^{\prime}\right) d \mathbf{f}^{\prime} \\
& +\sigma_{n}^{2} \mathbf{T}_{c}^{\prime}(\mathbf{f}, \mathbf{0})+\sigma_{n} \overline{\sigma^{0}}[C(\mathbf{f})+C(-\mathbf{f})]
\end{aligned}
$$

where the $c$ index indicates parameters related to the compressor filter, and $R_{h_{c}}$ and $\mathbf{T}_{c}^{\prime}$ are defined similar to $R_{h}$ and $\mathrm{T}^{\prime}$; see (17) and (24). $C(\mathbf{f})$ is given by

$$
\begin{aligned}
C(\mathbf{f}) & =F\left\{R_{h}(\mathbf{r}) R_{h_{c}}^{*}(\mathbf{r})\right\} \\
& =\int|H(\boldsymbol{\xi}+\mathbf{f})|^{2}\left|H_{c}(\boldsymbol{\xi})\right|^{2} d \boldsymbol{\xi} .
\end{aligned}
$$

From (32) one can by integration find the average image power

$$
\begin{aligned}
\bar{R}_{s}(\mathbf{0})= & \langle E\{s(\mathbf{r}) s(\mathbf{r})\}\rangle=2\left\{\left[\sigma_{n} R_{h_{c}}(\mathbf{0})\right]^{2}\right. \\
& +\int \bar{S}_{\sigma}(\mathbf{f}) \mathbf{T}(\mathbf{0}, \mathbf{f}) d \mathbf{f} \\
& \left.+2 \sigma_{n} R_{h_{c}}(\mathbf{0}) \overline{\sigma^{0}} R_{h}(\mathbf{0})\right\} .
\end{aligned}
$$

This again implies that the power to mean square ratio (PMR) of the image is

$\mathrm{PMR}=\frac{\bar{R}_{s}(\mathbf{0})}{\langle E\{s(\mathbf{r})\}\rangle^{2}} \bar{R}_{s}=$

$$
\frac{2+\mathrm{PMR}_{\sigma^{0}} \cdot\left(\frac{S}{N}\right)^{2}+4 \cdot\left(\frac{S}{N}\right)}{\left[1+\left(\frac{S}{N}\right)\right]^{2}}
$$

where

$$
\frac{S}{N}=\frac{\sigma^{0} R_{h}(\mathbf{0})}{\sigma_{n} R_{h_{c}}(\mathbf{0})} .
$$

\section{SIMPLIFYING APPROXIMATION}

One of the most widely used models of speckle noise in radar images is

$s(\mathbf{r})=\sigma^{0}(\mathbf{r}) \cdot \operatorname{sn}(\mathbf{r})$

where $\sigma^{0}(\mathbf{r})$ is the mean backscatter coefficient of the scene, and $s n(\mathbf{r})$ is a multiplicative speckle noise factor, and $\sigma^{0}$ and $s n$ are independent $[5,6,12]$. Equation (38) leads to

$\bar{R}_{s}(\mathbf{r})=\bar{R}_{\sigma^{0}}(\mathbf{r}) \cdot R_{s n}(\mathbf{r})$.

For a homogeneous area we have

$\bar{R}_{\sigma^{0}}(\mathbf{r})=$ const $=\left(\sigma^{0}\right)^{2}$

and therefore a comparison with (16) gives

$$
\begin{aligned}
R_{s n}(\mathbf{r})= & R_{h}(\mathbf{0})^{2}+\left|\mathbf{R}_{h}(\mathbf{r})\right|^{2}=\left.\left.\left|\int\right| h(\mathbf{a})\right|^{2} d \mathbf{a}\right|^{2} \\
& +\left|\int h(\mathbf{a}) h^{*}(\mathbf{a}-\mathbf{r}) d \mathbf{a}\right|^{2} .
\end{aligned}
$$

If (39) and (41) are then compared with (19) we see that (38) is only a useful model when $\bar{R}_{\sigma^{0}}(\mathbf{r})$ is slowly 
varying compared to the system impulse response function $h(\mathbf{r})$.

If, however, we can assume $\sigma^{0}$ is slowly varying, we find, with no additional assumptions, that

$$
\begin{aligned}
& S_{s n}(\mathbf{f})=\left.\left.\delta(\mathbf{f})\left|\int\right| H(\zeta)\right|^{2} d \zeta\right|^{2} \\
&+\int|H(\mathbf{f}+\zeta)|^{2}|H(\zeta)|^{2} d \zeta
\end{aligned}
$$

and, using $\circ$ as the convolution operator, we have

$$
\begin{aligned}
\bar{S}_{s}(\mathbf{f})= & \bar{S}_{\sigma^{0}}(\mathbf{f}) \circ S_{s n}(\mathbf{f}) \\
= & \left.\left.\bar{S}_{\sigma^{0}}(\mathbf{f})\left|\int\right| H(\zeta)\right|^{2} d \zeta\right|^{2} \\
& +\iint \bar{S}_{\sigma^{0}}\left(\mathbf{f}^{\prime}\right)\left|H\left(\mathbf{f}-\mathbf{f}^{\prime}+\zeta\right)\right|^{2} \\
& \times|H(\zeta)|^{2} d \mathbf{f}^{\prime} d \zeta \\
\simeq & \left.\left.\bar{S}_{\sigma^{0}}(\mathbf{f})\left|\int\right| H(\zeta)\right|^{2} d \zeta\right|^{2}+\int \bar{S}_{\sigma^{0}}\left(\mathbf{f}^{\prime}\right) d \mathbf{f}^{\prime} \\
& \cdot \int|H(\mathbf{f}+\zeta)|^{2}|H(\zeta)|^{2} d \zeta \\
= & \bar{S}_{\sigma^{0}}(\mathbf{f}) \cdot \mathbf{T}(\mathbf{0}, \mathbf{0})+\int \bar{S}_{\sigma^{0}}\left(\mathbf{f}^{\prime}\right) d \mathbf{f}^{\prime} \cdot \mathbf{T}^{\prime}(\mathbf{f}, \mathbf{0}) .
\end{aligned}
$$

In cases where the assumption is applicable (43) is found to be a major simplification. Firstly, only the power transfer function needs to be known, and, as was shown in (11), it can be estimated from the complex data.

Secondly, integration on both sides of (43) gives

$$
\begin{aligned}
\int \bar{S}_{s}(\mathbf{f}) d \mathbf{f} & =\int \bar{S}_{\sigma^{0}}\left(\mathbf{f}^{\prime}\right) d \mathbf{f}^{\prime}\left\{\mathbf{T}(\mathbf{0}, \mathbf{0})+\int \mathbf{T}^{\prime}(\mathbf{f}, \mathbf{0}) d \mathbf{f}\right\} \\
& =2 \cdot \mathbf{T}(\mathbf{0}, \mathbf{0}) \int \bar{S}_{\mathbf{\sigma}^{0}}\left(\mathbf{f}^{\prime}\right) d \mathbf{f}^{\prime}
\end{aligned}
$$

where the inverse Fourier transform of (24) has been used. Then using (43) and (44), $\bar{S}_{\sigma^{0}}(\mathbf{f})$ can be expressed in the closed form

$$
\begin{aligned}
\bar{S}_{\boldsymbol{\sigma}^{0}}\left(\mathbf{f}^{\prime}\right)= & \frac{1}{\mathbf{T}(\mathbf{0}, \mathbf{0})}\left\{\bar{S}_{s}(\mathbf{f})-\frac{\mathbf{T}^{\prime}(\mathbf{f}, \mathbf{0})}{2 \mathbf{T}(\mathbf{0 , 0})}\right. \\
& \left.\cdot \int \bar{S}_{s}\left(\mathbf{f}^{\prime}\right) d \mathbf{f}^{\prime}\right\} .
\end{aligned}
$$

Note, that $\mathbf{T}(\mathbf{0 , 0})$ and $\mathbf{T}^{\prime}(\mathbf{f}, \mathbf{0})$ are determined solely by the power transfer function, $|H(\mathbf{f})|^{2}$, [see (29) and (30)], which can be estimated from the complex image data (11).

\section{CONCLUSION}

The model presented shows that the average power spectrum of the complex SAR image is not dependent on scene homogeneity. Therefore, estimation of the power transfer function $|H(\mathbf{f})|^{2}$ can be performed using nonhomogeneous images. This is in contrast to extracting similar spectral information from power images, where scene homogeneity is essential. For nonhomogeneous radar scenes, the relation between the radar scene frequency spectrum and the radar power image spectrum is not given by a simple transfer function, but by a linear integral equation. This means that one frequency in the radar scene will give rise to a set of frequencies in the power image, as expressed by (25). To define the integral equation completely, the complex IPR (impulse response function) of the SAR system must be known. Usually this can only be achieved through calibration against reference reflectors.

If it can be assumed that the scene properties are slowly varying compared with the width of the IPR, then only the power transfer function needs to be known. As mentioned, the estimation of this function can be accomplished from the complex SAR data, whether the scene is homogeneous or not. In this quasi-stationary situation an inversion can be performed, so that the radar scene spectrum-which is of interest-can be expressed in a closed form involving the observed radar image spectrum and the system power transfer function.

\section{APPENDIX A BASIC THEORY OF NONSTATIONARY PROCESSES}

In this Appendix some definitions of the theory of nonstationary processes relevant to this paper is reviewed. For simplicity in notation, the processes are assumed to be one-dimensional. Nonstationary processes is also treated by Papoulis [13].

The correlation function of $x$ and $y$ is

$R_{x y}\left(t_{1}, t_{2}\right)=E\left\{x\left(t_{1}\right) y^{*}\left(t_{2}\right)\right\}$.

For a linear system with input $x(t)$, IPR $h(t)$, and output $y(t)$, we have

$y(t)=\int x(t-a) h(a) d a$

and hence

$$
\begin{aligned}
R_{y y}\left(t_{1}, t_{2}\right) & =\iint h(a) h^{*}(\beta) R_{x x}\left(t_{1}-a, t_{2}-\beta\right) d a d \beta \\
& =h\left(t_{1}\right) \circ h^{*}\left(t_{2}\right) \circ R_{x x}\left(t_{1}, t_{2}\right)
\end{aligned}
$$

where $\circ$ indicates convolution.

We now introduce the two-dimensional Fourier transformation of $R\left(t_{1}, t_{2}\right)$

$$
\begin{aligned}
S\left(f_{1}, f_{2}\right)= & \iint R\left(t_{1}, t_{2}\right) \\
& \times \exp \left[-j 2 \pi\left(f_{1} t_{1}+f_{2} t_{2}\right)\right] d t_{1} d t_{2} .
\end{aligned}
$$

We find from (A3) and (A4) that

$S_{y y}\left(f_{1}, f_{2}\right)=H\left(f_{1}\right) H^{*}\left(-f_{2}\right) S_{x x}\left(f_{1}, f_{2}\right)$.

Even though $R\left(t_{1}, t_{2}\right)$ is nonstationary, one can in general define a space or time averaged correlation function. Its form will depend on the observed signal, as to whether it is a finite energy signal or finite power signal. For the finite power signal we define 
$\bar{R}(\tau)=\langle R(t+\tau, t)\rangle_{t}=\lim _{T \rightarrow \infty} \frac{1}{2 T} \int_{-T}^{T} R(t+\tau, t) d t$

where \langle\rangle indicates the time (or space) average operator. The average power density is derived from

$\bar{S}(f)=\int \bar{R}(\tau) \exp (-j 2 \pi f \tau) d \tau$.

Combining (A3) and (A6) gives

$\bar{S}_{y y}(f)=|H(f)|^{2} \bar{S}_{x x}(f)$.

\section{ACKNOWLEDGMENT}

I want to thank Farida Dahl for her help in preparing the manuscript. Also, thanks are expressed to Jørgen Dall and Professor Preben Gudmandsen for reviewing the article and providing constructive suggestions.

\section{REFERENCES}

[1] Ulaby, F.T., Moore, R.K. and Fung, A.K. (1982) Microwave Remote Sensing, Active and Passive. New York: Addison-Wesley, 1982, ch. 9.

[2] Mitchell, R.L. (1974) Models of extended targets and their coherent radar images. Proceedings of the IEEE, 62, 6 (June 1974), 754-758.

[3] Goodman, J.W. (1975) In Statistical Properties of Laser Speckle Patterns in Laser Speckle and Related Phenomena, J.C. Dainty, Ed.

New York: Springer-Verlag, Topics in Applied Physics, Vol. 9, 1975.

[4] Jao, J.K. (1984)

Amplitude distribution of composite terrain radar clutter and the $K$ distribution.

IEEE Transactions on Antennas and Propagation, AP-32, 10 (Oct. 1984), 1049-1062.
[5] Goldfinger, A.D. (1982)

Estimation of spectra from speckled images.

IEEE Transactions on Aerospace and Electronic Systems, AES-18, 5 (Sept. 1982), 675-681.

[6] Piau, P., and Gray, L. (1975) SAR imaging of the sea surface during the "PROMESS" Experiment.

In Proceedings of EARLSeL, ESA Symposium "European Remote Sensing Opportunities" (Strasbourg, ESA SP-233, May 1985), pp. 131-138.

[7] Madsen, S.N. (1986)

Speckle theory: modeling, analysis, and applications related to synthetic aperture radar data.

$\mathrm{Ph} . \mathrm{D}$. Thesis, LD 62, Electromagnetics Institute, Technical University of Denmark, ch. 5.

[8] Harger, R.O. (1970) Synthetic Aperture Radar Systems, Theory and Design. New York: Academic Press, 1970, p. 85.

[9] Brown, W.M. (1967) Synthetic aperture radar.

IEEE Transactions on Aerospace and Electronic Systems, AES-3 (Mar. 1967), 217-229.

[10] Reed, I.S. (1962)

On a moment theorem for complex Gaussian processes. IRE Transactions on Information Theory (Apr. 1962), 194-195.

[11] Harrington, R.F. (1968)

Field Computations by Moment Method.

New York: Macmillan, 1968, sec. 1.3.

[12] Frost, S.F., Stiles, A.S., Shanmugan, K.S., and Holtzman, J.C. (1982)

A model for radar images and its application of adaptive digital filtering of multiplicative noise.

IEEE Transactions on Pattern Analysis and Machine Intelligence, PAMI-4, 2 (Mar. 1982), 157-166.

[13] Papoulis, A. (1965)

Probability, Random Variables and Stochastic Processes. New York: McGraw-Hill, ch. 12.

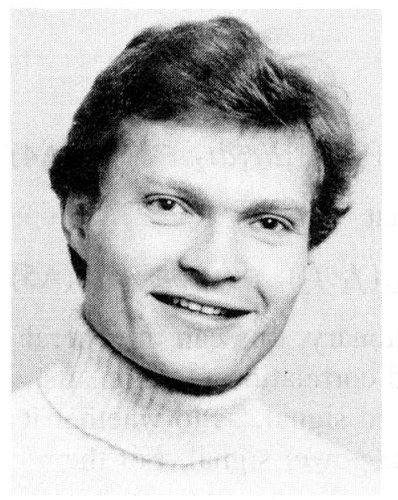

Søren Nørvang Madsen received the M.Sc. degree in electrical engineering and electrophysics from the Technical University of Denmark in 1982 with a thesis on a computer controlled nuclear magnetic resonance (NMR) spectrometer. He received the $\mathrm{Ph} . \mathrm{D}$. degree in electrical engineering in 1987 with a thesis on properties of synthetic aperture radar images.

He has been with the Electromagnetics Institute, Technical University of Denmark, since 1982. He has been working on research within the field of basic statistics of synthetic aperture radar images, optimal post filtering, and SAR preprocessor developments. He has participated in several field experiments using radar and radiometer for remote sensing of sea ice and oil pollution. Since 1984 he has been an Associate Professor at the Electromagnetics Institute, working primarily with digital signal processing and radar theory. He is currently Project Manager on a coherent radar project concerned with SAR, weather radar, and pulse-Doppler radars. 